GA-A21709

\title{
RESULTS OBTAINED USING THE PELLET CHARGE EXCHANGE DIAGNOSTIC ON TFTR
}

\begin{abstract}
by
J.M. McCHESNEY, H.H. DUONG, ${ }^{*}$ R.K. FISHER, D.K. MANSFIELD, ${ }^{\dagger}$ S.S. MEDLEY, ${ }^{\dagger}$ P.B. PARKS, M.P. PETROV ${ }^{\ddagger}$ and A.L. ROQUEMORE ${ }^{\dagger}$

This is a preprint of a paper to be presented at the Tenth Topical Conference on High Temperature Plasma Diagnostics, May 8-12, 1994, Rochester, New York, and to be printed in REVIEW OF SCIENTIFIC INSTRUMENTS.
\end{abstract}

\author{
Work supported by \\ U.S. Department of Energy \\ under Grant No. DE-FG03-92ER54150 \\ and Contract No. DE-AC02-76CHO3073 \\ * University of California at Irvine. \\ † Princeton Plasma Physics Laboratory. \\ ${ }_{\ddagger}$ A.F. Ioffe Physical-Technical Institute.

\section{GENERAL ATOMICS PROJECT 3937 MAY 1994}




\section{DISCLAIMER}

This report was prepared as an account of work sponsored by an agency of the United States Government. Neither the United States Government nor any agency thereof, nor any of their employees, makes any warranty, express or implied, or assumes any legal liability or responsibility for the accuracy, completeness, or usefulness of any information, apparatus, product, or process disclosed, or represents that its use would not infringe privately owned rights. Reference herein to any specific commercial product, process, or service by trade name, trademark, manufacturer, or otherwise, does not necessarily constitute or imply its endorsement, recommendation, or favoring by the United States Government or any agency thereof. The views and opinions of authors expressed herein do not necessarily state or reflect those of the United States Government or any agency thereof. 


\section{DISCLAIMER}

Portions of this document may be illegible in electronic image products. Images are produced from the best available original document. 


\section{ABSTRACT}

Experiments are underway on TFTR to measure the confined alpha particle distribution functions using small low-Z pellets injected into the plasma. ${ }^{1}$ Upon entering the plasma, the pellet ablates, forming a plasma ablation cloud, elongated in the magnetic field direction, that travels alongside the pellet. A small fraction of the fusion produced $3.5 \mathrm{MeV}$ alpha particles incident on the cloud are converted to helium neutrals. By measuring the resultant helium neutrals escaping from the plasma by means of a mass and energy resolving charge exchange analyzer, the energy distribution of the alpha particles incident on the cloud can be inferred. Preliminary experiments to observe neutrals from the $100-1000 \mathrm{keV}{ }^{3} \mathrm{He}$ tail produced during ICRF minority heating experiments were successful. However, no significant alpha particle signals have been observed during D-T operation on TFTR. We attribute this lack of signal to stochastic toroidal field ripple loss in the outer regions of the plasma. We are studying ways to improve the pellet penetration so that the pellet penetrates into the central regions of the plasma where ripple induced losses are small and the alpha population is high. 


\section{INTRODUCTION}

Fusion ignition requires that the alpha particles created by the deuteriumtritium (D-T) reaction deposit a large fraction of their energy in the plasma before they are lost. Various alpha particle diagnostics have been reviewed. ${ }^{2}$ Fisher et al. ${ }^{1}$ have advocated the pellet charge exchange (PCX) method to measure the confined alpha particle velocity distribution function. Upon entering the plasma, the pellet material ablates forming an ablation cloud elongated along the field lines, that travels along with the pellet, as shown schematically in Fig. 1. As the ablated material moves away from the pellet surface, it is ionized to successively higher charge states until a helium-like charge state is attained, where the ionization potential jumps by an order of magnitude or more. A large region of the ablation cloud is dominated by this helium-like state. This region provides a high density target with which the alpha particles can charge exchange, either by the double charge exchange reaction, or by two sequential single charge exchange reactions. The resultant helium neutrals exit the plasma, and are detected by a neutral particle analyzer (NPA). If the line integral density experienced by particles passing through the ablation cloud is sufficiently large, then the fraction of the incident alphas exiting the cloud as neutrals approaches $F_{0}^{\infty}(E)$ (equilibrium fraction), which is independent of the cloud parameters. The signal $(d N / d t)$ measured at each analyzer channel is given by,

$$
\frac{d N}{d t}=\frac{d n_{\alpha}}{d E}\left[F_{0}^{\infty}(E) v_{\alpha} A_{\text {cloud }}\left(\frac{\Omega}{4 \pi}\right) \eta \Delta E\right]
$$

where $A_{\text {cloud }}$ is the observed cloud area, $(\Omega / 4 \pi)$ the solid angle factor, $\eta$ the stripping foil efficiency, and $\Delta E$ the energy resolution of the channel. Equation (1) is only 
strictly correct when the Larmor radius is much larger than the cloud radius. When these dimensions are comparable, it is possible for an alpha to make several passes through the cloud, thereby enhancing the neutralization fraction. The number of passes goes as $\left(\cos \theta_{p}\right)^{-1}\left(\theta_{p}=\right.$ pitch angle), and the velocity goes $\left(\cos \theta_{p}\right)$. So to first order these two effects cancel in Eq. (1). A separate paper discussing these effects in detail is presently in preparation. In principle, the helium-like charge state of any low $Z$ pellet can be used. Lithium was selected because it has the highest theoretically predicted signal levels, and was found to possess desirable wall conditioning properties on TFTR.

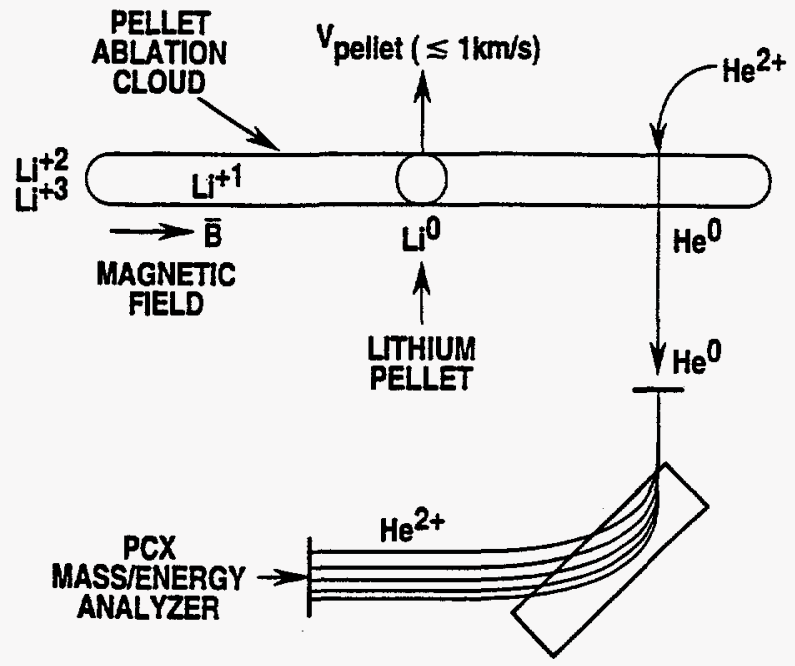

Fig. 1. Schematic showing the experimental technique. 


\section{EXPERIMENTAL LAYOUT}

The PCX diagnostic on TFTR consists of a pellet injector which injects the impurity pellet in the midplane along a major radius, and a neutral particle analyzer (NPA) with a sightline at a selectable toroidal angle to the pellet trajectory of $2.75^{\circ}$, $13^{\circ}$, or $18.37^{\circ}$. The pellet injector is capable of injecting pellets with velocities in the range $400-700 \mathrm{~ms}^{-1}$. The pellets are cylindrical, typically $1.7 \mathrm{~mm}$ (diameter) by $3 \mathrm{~mm}$ (length) and contain $7.3 \times 10^{19}$ atoms.

The analyzer sightline was fixed during a day's run period. In the work reported here, the NPA was positioned at the $2.75^{\circ}$ angle which viewed the pellet ablation cloud about $12 \mathrm{~cm}$ toroidally from the pellet. The NPA is of the $E \| B$ type and is capable of simultaneous mass and energy analysis. ${ }^{3}$ The analyzer has eight channels with $\mathrm{ZnS}(\mathrm{Ag})$ scintillators; light emission is measured by photomultiplier tubes. The expected signal levels ${ }^{1}$ were high enough that the PMTs were operated in an analog mode rather than a pulse counting mode. The NPA was situated inside the TFTR test cell, and was therefore subjected to the full neutron flux during high power D-T operation. To shield the scintillators and PMT's, a radiation shield consisting of 4 in. of lead inside 6 in. of borated polyethelene almost completely surrounded the analyzer. PMT/scintillator combinations were placed both inside and outside the shield to measure the attenuation. The shield attenuation was $150 \mathrm{x}$ for $14 \mathrm{MeV}$ neutrons, and 500x for $2.5 \mathrm{MeV}$ neutrons. 


\section{RECENT RESULTS ON TFTR}

In the experiments conducted on TFTR, the pellet was unable to penetrate to the plasma core under all conditions. Penetration was determined by measuring the pellet cloud light emission using a linear diode array situated on top of the machine. The penetration was increased by injecting two pellets, with a selectable interval (5-100 ms) between them. The first pellet rapidly lowered the global electron temperature by a factor of two or more (depending on the pellet size). However, the slowing down time of the fast ions is much larger. In the cooled plasma, the slowing down time of a $1 \mathrm{MeV}$ alpha is $30-40 \mathrm{~ms}$. So by keeping the time interval between the two pellets less than this value, it was still possible to make a useful measurement of the energetic particle distribution.

During discharges heated by RF power only (no beam heating), the first pellet penetrated to $r / a \sim 0.4$, but the second pellet penetrated all the way to the plasma center. In neutral beam heated plasmas, however, the first pellet only penetrated to $r / a \sim 0.6$ and the second pellet penetrated less than $10 \mathrm{~cm}$ further. During neutral beam heated discharges, the pellets were generally fired 100-200 ms after the beams were turned off. This allowed $T_{e}$ and $n_{e}$ to decay somewhat, while the confined alpha distribution was only expected to decay modestly.

During recent ${ }^{3} \mathrm{He}$ minority $\mathrm{RF}$ heating experiments, measurements of energetic ${ }^{3}$ He tails were obtained. Figure 2 shows data taken during TFTR shot \#73741. This was an RF only discharge, no heating beams were used, and the total RF power was 4.2 MW. The top three traces show the plasma current, the RF power, and the line integral plasma density. On an expanded time scale, the lower two traces show the signal from one of the analyzer channels, and the $\mathrm{Li}^{+}$light measured by a detector 

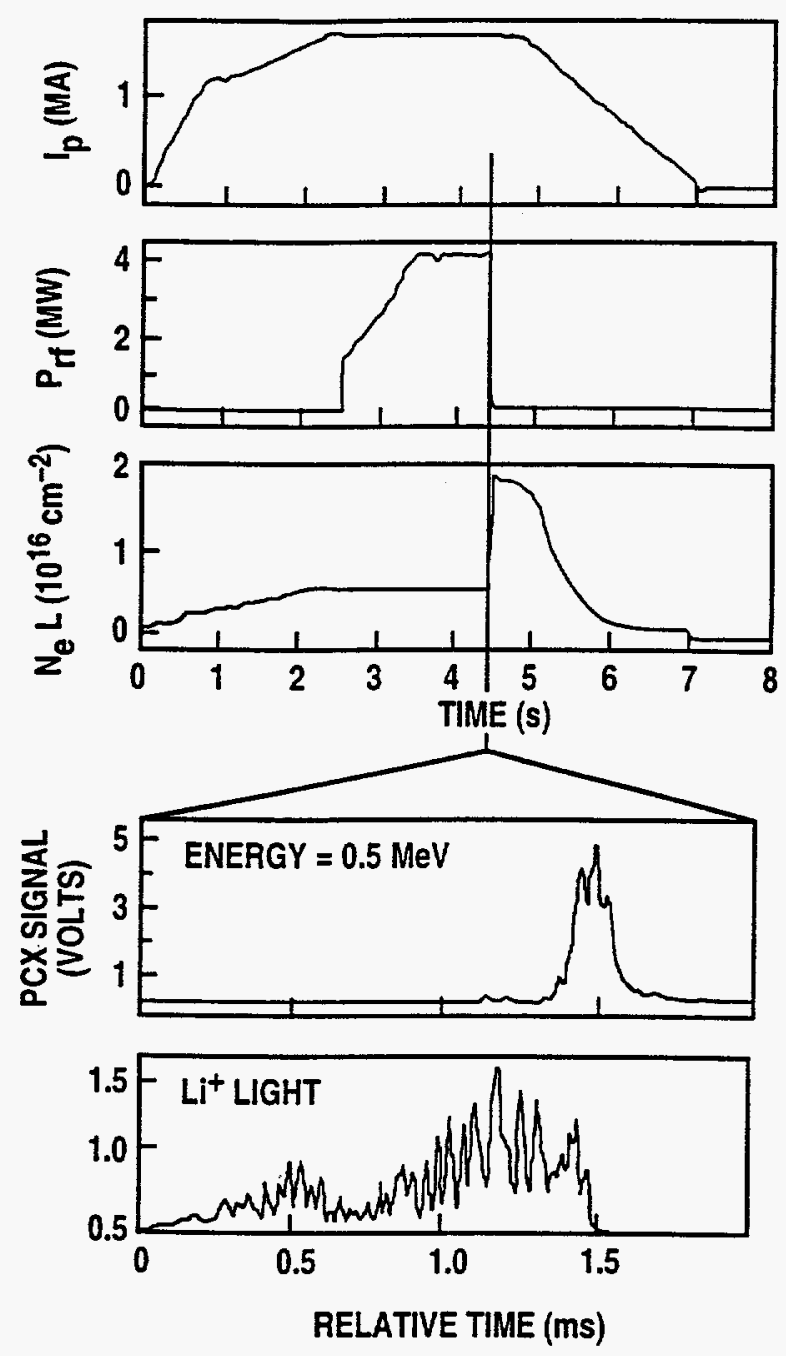

Fig. 2. Waveforms for TFTR shot \#73741. (a) Plasma current. (b) RF power. (c) Line integral plasma density at $R=2.68 \mathrm{~m}$. (d) Raw data from $0.5 \mathrm{MeV}$ analyzer channel. (e) $\mathrm{Li}^{+}$light at $548.5 \mathrm{~nm}$.

which views the pellet light along the analyzer sightline. This was the second pellet of a pair that was injected as the RF power was being turned off. The pellets were injected at $\mathrm{t}=4.45 \mathrm{~s}$ and $\mathrm{t}=4.47 \mathrm{~s}$. The spectra obtained for these two pellets are shown in Fig. 3. The RF resonant layer was at $2.70 \mathrm{~m}$. The first pellet penetrated to $R=3.10 \mathrm{~m}$ while the second penetrated to $\mathrm{R}=2.80 \mathrm{~m}$ and was closer to the 


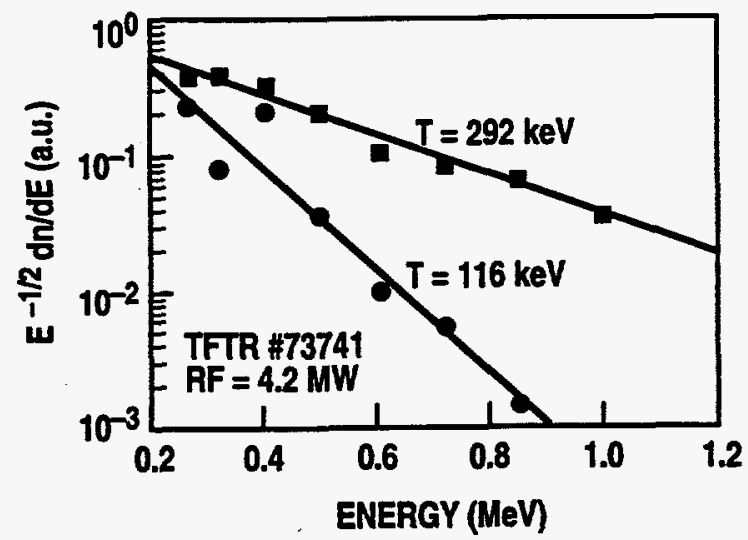

Fig. 3. ${ }^{3} \mathrm{He}$ minority heating experiment (TFTR shot \#73741). Pellet 1 (circles), pellet 2 (squares).

$\mathrm{RF}$ resonant surface. This is reflected in the higher temperature and larger values of $d n / d E$.

Measurements were also made during hydrogen minority heating experiments. These measurements were made both actively (PCX), and passively, using charge exchange on hydrogen-like carbon $\left(C^{5+}\right)$, found in the core of TFTR. ${ }^{4}$ Figure $4(\mathrm{a})$ shows data obtained using the PCX method, during shot \#75035. The pellet penetrated to $3.0 \mathrm{~m}$ and the $\mathrm{RF}$ resonant layer was at $2.80 \mathrm{~m}$, so the pellet penetrated to within $20 \mathrm{~cm}$ of the resonant layer. Figure 4(b) shows passive data taken during shot \#75214. The RF heating power was 4.65 MW during the passive measurements and 4.0 MW during the active measurements. Within the limits of experimental error, the two measurements agree.

It should be noted that the vertical axes in Figs. 4(a) and 4(b) are arbitrary and were obtained by different means. The active measurement was obtained by digitizing the dc current from the PMT's during the pellet lifetime, while the passive data were determined by counting individual pulses over a period of one second. The passive count rates were about $10^{2}$ to $10^{3}$ counts/s/channel while the active count rates were $10^{5}$ to $10^{7}$ counts/s/channel. Although the active count rates were 

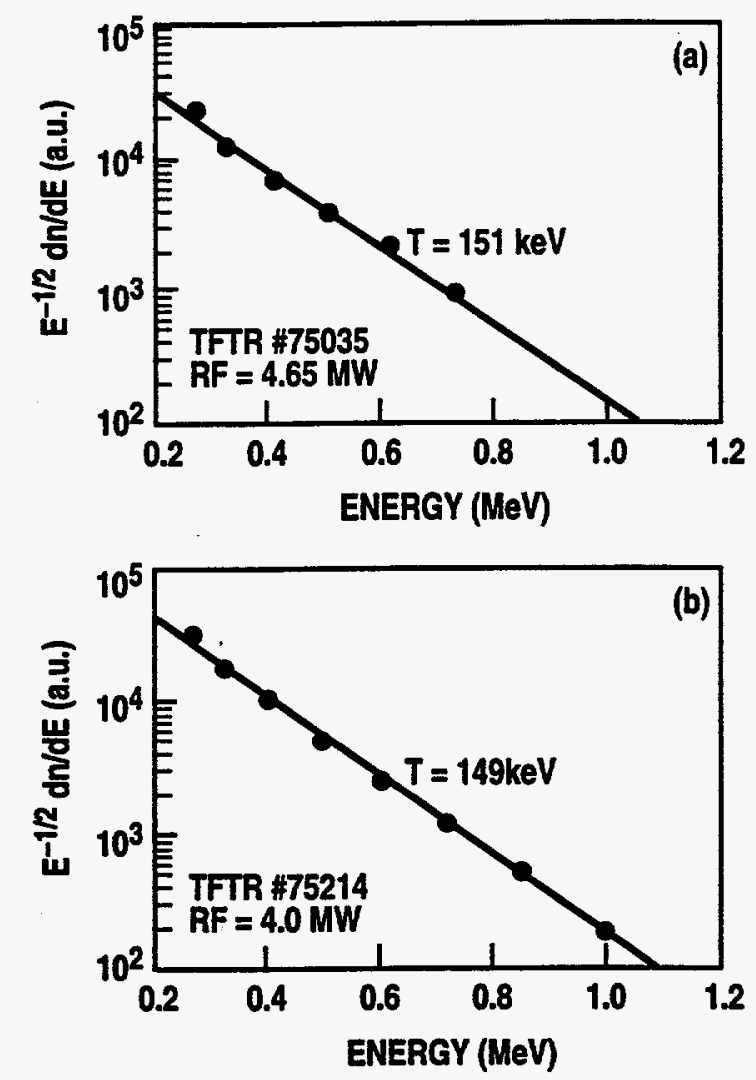

Fig. 4. Hydrogen minority heating experiments. (a) Active measurements (TFTR shot \#75035). (b) Passive measurement (TFTR shot \#75214).

considerably higher than the passive count rates, they were still about 100 times lower than expected from theory for both $\mathrm{H}$ and ${ }^{3} \mathrm{He}$ ICRF heating experiments. The reasons for this are unclear. We have considered many possible explanations for the smaller than expected signal rate, but have not as yet found one that is consistent with all the data.

Two D-T shots have been provided for testing the PCX diagnostic. [Shot numbers 73254 (lithium pellet) and 73255 (boron pellet).] No significant alpha particle signals were observed during these discharges. The analyzer was configured to view the alpha slowing down spectrum, with the energy range set to $0.5-2.0 \mathrm{MeV}$. The most likely explanation for our lack of alpha signal is stochastic ripple diffusion, which can significantly deplete the fast ion population in the outer regions of TFTR. ${ }^{5}$ The 
pellet could not penetrate to regions where stochastic ripple loss was unimportant $(r / a<0.5)$. 


\section{CONCLUSIONS}

The PCX diagnostic has been tested on TFTR and has measured both energetic ${ }^{3} \mathrm{He}$ and $\mathrm{H}$ neutrals arising from the RF heated ion tails interacting with lithium pellet clouds. Most encouragingly, the tail temperatures measured for the RF generated $\mathrm{H}$ tails were in good agreement with passive measurements not involving pellets. No significant alpha particle signals were observed during D-T operation. The most likely explanation for this lack of signal is stochastic ripple loss of the alpha particles. In beam heated discharges, the pellet was unable to penetrate to the core regions where ripple losses were unimportant. This contrasts with $\mathrm{RF}$ heated discharges where the pellet penetrated to the plasma center.

In order to reach regions where stochastic ripple loss is not a problem, we plan to increase pellet penetration, by using larger pellets and by increasing the pellet velocity. Signal levels can also be improved if we switch from viewing ripple trapped particles to looking at passing particles, which may be possible for future machines.

The PCX technique should be applicable to ITER, but will likely be limited to studies outside $r / a \simeq 0.5$ due to poor pellet penetration and re-ionization of neutrals leaving the plasma. 


\section{REFERENCES}

1 R.K. Fisher, J.S. Leffler, A.M. Howald, and P.B. Parks, Fusion Technol. 13, 536 (1988).

2 S.J. Zweben, Rev. Sci. Instrum. 57, 1723 (1986).

3 A.B. Izvozchikov, A.V. Kuhdoleev, M.P. Petrov, S.Ya. Petrov, S.S. Kozlovskij, S. Corti, and A. Gondhalekar, JET Report JET-R(91)12, (1991).

4 A. Gondhalekar, A. Korotkov, S. Corti, A.V. Khudoleev, M.R. O'Brien, M.P. Petrov, D. Start, A. Start, and C.D. Warrick, in Proc. IAEA Technical Committee Meeting on Alpha Particle Physics in Fusion Research, May 10-14, 1993, Trieste, Italy.

5 R.J. Goldston, R.B. White, A.H. Boozer, Phys. Rev. Lett. 47, 647 (1981). 


\section{ACKNOWLEDGMENT}

This is a report of work supported by the U.S. Department of Energy under Grant No. DE-FG03-92ER54150 and Contract No. DE-AC02-76CHO3073. 